\title{
Some biolinguistic remarks
}

\author{
Michael Brody \\ University College London \\ mbbrody@gmail.com
}

\begin{abstract}
After comments with laudatory and clarifying intent on the Chomskyan revolution, I make some critical remarks on Eörs Szathmáry's views on the evolution of language and its relation to the brain. This is followed by a brief sketch of the theory of one-dimensional syntax in the biolinguistic context, leading to the conclusion that the item-organizing specificity of human language may not be due to a qualitative change in narrow syntactic ability. This unique property may instead be a consequence of the possession of an interpretive module capable of parallel processing of numerous simple onedimensional syntactic structures - which individually have no more configurational complexity than birdsong.
\end{abstract}

Keywords: Chomskyan revolution; evolution of language; one-dimensional syntax; recursion; center embedding

As a result of unfortunate early popularization and misplaced emphasis, many people still think of Chomsky as the person whose main scientific discovery was deep structure and transformational grammar. ${ }^{1}$ But the concept of transformation has already been used in linguistics before (see Dékány 2019), and deep structure has not been part of the theory since the 1990s. ${ }^{2}$ To consider Chomsky the theorist of deep structure is not more adequate than considering Darwin as the theorist of gemmules, the particles that were supposed to be transferred to the gonads by every body part to account for inheritance in his version of the pangenesis theory.

${ }^{1}$ This is a translation with some edits of a paper written for a Hungarian volume (Kenesei 2019) prepared for the occasion of Noam Chomsky's 90th birthday. I am grateful to an anonymous reviewer of the English version for a careful reading and very helpful comments.

${ }^{2}$ I showed at the turn of the ' 80 s and '90s that deep structure was an unnecessary construct within the principles and parameters theory (Brody 1987; 1992; 1993). Later, Chomsky himself rejected the postulate on essentially the same grounds (Chomsky 1995). 
In fact, as highlighted by Dékány in her excellent review, Chomsky's indisputable achievement is that he has freed linguistics and related disciplines from the straitjacket of behavioral science. He has consistently argued for and represented in his work the approach that has long been taken for granted in the more advanced natural sciences. This liberation basically meant two things. On the one hand, it meant rejecting the dogma that the properties of the mind are beyond the scope of science. Just as physics is not the science of meter readings, as he famously noted, the science of linguistics should not be limited either to describing and classifying the external linguistic products of the mind.

On the other hand, just as in physics the Cartesian mechanistic worldview has become untenable after Newton's surprising but highly successful abstract theories, after the Chomskyan revolution the naive mechanistic behaviorist approach became uninteresting and untenable in cognitive psychology and linguistics. This involved first and foremost the restoration of the legitimacy of abstraction and of the freedom of scientific theorizing. Theorizing is not possible without abstractions. Incidentally, the word might mislead. Despite appearances, valid abstract theoretical constructs are actually closer to reality than observable facts - a perhaps surprising statement that is a straightforward consequence of construing science as a search for truth. At least in the case of genuinely explanatory theories observable facts can typically be accounted for by abstract rule systems only through a complex chain of deductive reasoning. Understanding this point about the status of abstractions leads not to a dismissal, but to a proper appreciation of the importance of observable facts as the anchor points of our scientific grasp of what is real.

Just like, for example, Newton's second law abstracts away from friction, the linguist must distinguish the linguistic knowledge grammar is a model of and the use of language. Of course, there are many difficult questions about what this abstraction actually is, where the dividing line lies, and how many different kinds of distinctions it is right and proper to make between knowledge and use and between grammatical correctness and acceptability. But the unsupported idea that regularly resurfaces, that these distinctions, like the concept of the deep structure, can be left by the wayside seems best interpreted as a rejection of abstraction, as evidence of the resilience of behaviorist thinking.

With the revival of genuine natural science standards, linguistics has quickly developed a level and atmosphere that is not always characteristic of social sciences, in which it is not possible to imitate expertise only with lexical knowledge or good verbal skills. As Chomsky has memorably said 
at some point in his MIT lectures during the early eighties, uncharacteristically using strong language: due to the complexity of the issues raised, "bullshitting" becomes extremely difficult - a sign of a certain maturity of the field. Lack of competence or contribution, lack of desire or ability to engage with real scientific issues is necessarily quickly revealed.

So Chomsky can legitimately be compared to Newton in that he has made abstract theoretical work legitimate and standard with successful theories in his own field. In another respect, he is, I think, closer to Darwin than to Newton. His central and lasting contribution is not one of the rule systems he proposed, however insightful and important these may be. It is rather the creation of a long-term paradigm that is based on a simple but powerful basic argument. Just as in life-sciences, the idea of natural selection provides the framework of thought that no significant biological theory can ignore, in the same way the poverty of the stimulus argument inescapably restricts and organizes linguistic theory construction. Serious work cannot ignore the consequences of these basic organizing concepts.

It follows from the poverty of the stimulus argument that, apart from abnormal or special cases, the system of universal grammar, which carries the common features of natural languages, is inheritably present in the minds of human language users. In addition, properties of individual natural languages that are not the consequence of universal grammar must be learnable during ontogenetic development. The basic question is, therefore, what the precise details of universal grammar are, what linguistic phenomena belong to it and to what extent, and what are those linguistic phenomena that we can rationally assume can be learned with the help of universal grammar and other cognitive and non-cognitive human abilities.

The postulation of an innate universal grammar, of course, raises many further interesting and important questions. Two of these issues are how this mental ability is realized in the brain and what evolutionary steps we need to assume were necessary for its development.

\section{2.}

Szathmáry $(2001 ; 2002 ; 2018)$ and Fedor et al. (2009; 2010) reviewed the problems of the brain structures and the evolution of language. Their discussion essentially ignores the distinction between universal grammar and individual natural languages, the difference between the innate and the acquired linguistic knowledge. Furthermore it shows no evidence of awareness of the distinction between narrow and broad linguistic abilities, the distinction between language and human specific linguistic abilities and 
those which are neither human nor language specific. The absence of these distinctions significantly constrains the potential value of their ideas. The evolution of inherited knowledge is not the same question as the evolution of some - domain-specific or not - learning ability. Furthermore, if the development of a non human-specific ability that is prerequisite for human language is at issue, there are many and radically different kinds of these from the expansion of vocabulary through the motor control of phonemes to pragmatic competence. We should therefore decide what natural classes of abilities are under discussion and how they differ from the capabilities of other animals in the same domain before we can start to think effectively, and not just in generalities, about how change could have taken place. '[...] treating 'language' as a monolithic whole both confuses discussions of its evolution and blocks the consideration of useful sources of comparative data" (Fitch et al. 2005, 181).

Fedor et al. (2009; 2010) suggest that their idea of coevolution of language with the theory of mind, teaching, tool use and co-operation is supported by the fact that people with autism have deficiencies in the areas of the theory of mind, communication and language. If anything, the autistic spectrum provides a counter-argument. Those with well-functioning autism "appear to have a good vocabulary and a sophisticated command of the language system" (Vicker 2009), while their theory of mind and social abilities are much less developed (e.g., Vicker 2009; Attwood 2007). If in autism damage to language and social skills were necessarily to have a common origin, well-functioning autism could not exist. However, this is of less importance here than the fact that if we wake up tomorrow to find that the rules of grammar are completely different, say the complete opposite of what we today think they are, not a single letter would need to be changed in this theory of coevolution. It is not optimal to think about human linguistic capacity ignoring its basic structure and properties (like structure-dependency, c-command, mirror-principle, etc.). It is as if someone would look at the development of the vertebrate's eyes, considering it merely as a photosensitive organ, ignoring all of its other major characteristics. If we try to describe the evolution of the human eye in this way, ignoring its specific qualities and structure, however well-informed we are in other respects, we will not be able to grasp the difference between an ancient photosensitive cell cluster and our eyes. So the evolutionary path between them will also by necessity remain undiscovered.

As for the brain structures of grammar, Fedor et al. use the rather unfortunate term of "language amoeba". They write that this "expresses the plasticity of the language". We find no explanation of how we should 
understand the supposed plasticity of language, just the statement that this "metaphor $[\ldots]$ also calls attention to the fact that a large part of the human brain is apparently a potential habitat for it" $(2009,4)$. In contrast to what the paragraph implies, this phenomenon of course has to do with the plasticity of the brain and says little about language. The logic here is parallel to that of the following strange assertion: By saying that the diamond can be easily kneaded into any shape, I 'also' refer to the fact that it can be located anywhere in a piece of plasticine.

It is difficult for the linguist to understand what Fedor et al. might mean when they speak about the plasticity of the language. The principles of universal grammar do not seem to be malleable at all. There is no language where the linearly first (e.g., Has the man who _ eaten must leave now?) and not the structurally prominent auxiliary (Must the man who has eaten _ leave now?) is moved if a yes-no question is created, as in English, by the fronting of its auxiliary verb. There is no such thing as massaging the principle of c-command in a sentence, so that a question word originating in the embedded sentence cannot land in the main sentence as in Who did she know Peter liked _ (from She knew Peter liked who) but instead the question word originating in the main sentence lands in the embedded clause as in _ Knew who Peter liked Mary (from Who knew Peter liked Mary).

So by plasticity of language, Fedor et al. $(2009,4)$ must actually mean the plasticity of the brain. They write: "Components of language (as well as those of other capacities) get localized during development somewhere in any particular brain in the most functionally "convenient" parts available (cf. Karmiloff-Smith 2006). Language is just a certain activity pattern of the brain that finds its habitat, like an amoeba in a medium". Choosing the most favorable habitat is not a particularly amoeba-specific feature - the metaphor is therefore not only mistaken and misleading, but unjustified even from this rather specific point of view. Also, Karmiloff-Smith does not write about the localization of language at all in the work quoted by Fedor et al. Her subject is that mental modules are formed during development, and the innateness of a module does not entail that there is a distinct set of genes responsible only for that module. It might follow from her arguments that the brain internal location of a particular module, such as language, is not necessarily fully genetically determined, but it does not follow at all that lack of genetic determination of brain location is necessarily or even typically the case. The lack of the basic genetic determination of localization does not follow either from the observation that "the localization of language components in the brain is extremely plastic" (ibid., 3), since 
genes can not only fix the topological coordinates in the brain directly, but by making other properties necessary, they will typically have different context-sensitive implicational consequences during ontogenesis, which may also pertain to or determine ultimate brain location.

Another logically dubious claim relating to the plasticity of the brain is the following: "[...] a very large part of the human brain can process linguistic information, including syntactical operations. This means that there is no fixed macro-anatomical structure that is exclusively dedicated to language" (ibid., 17). The argument is essentially parallel to the following non-sequitur: "On the basis of the genetics of a particular plant, we cannot predict the location of its flower, the flower with its characteristic functions can grow on various parts of the plant. It follows that the flower has no 'fixed macro anatomical structure' that is dedicated to whatever functions a flower has, like spore production etc." Clearly, it does not follow.

Fedor et al. continue: "This further suggests that there is some statistical connectivity feature of a large part of the human brain that renders it suitable for linguistic processing." This suggestion may (or may not) be true in a sense that is irrelevant here, where it is not meant to question the fact that language involves a mental organ with fixed macro anatomical structure and characteristic functions and properties of its own. In contrast to Fedor et al.'s assertion, it is clear that the plasticity of the brain in no way necessarily contradicts the possible existence of genetically encoded language module(s) with task specific dedicated structure(s) that develop during ontogeny.

\section{3.}

Hauser et al. (2002) and Fitch et al. (2005) define the narrow linguistic ability as that subpart of the broad linguistic ability which is both language-specific and specific to humans:

"[...] we denoted 'language' in the broad sense, including all of the many mechanisms involved in speech and language, regardless of their overlap with other cognitive domains or with other species, as the 'faculty of language in the broad sense' or FLB. This term is meant to be inclusive, describing all of the capacities that support language independently of whether they are specific to language and uniquely human. Second, given that language as a whole is unique to our species, it seems likely that some subset of the mechanisms of FLB is both unique to humans, and to language itself. We dubbed this subset of mechanisms the faculty of language in the narrow sense (FLN)." $\quad$ (Fitch et al. 2005, 181) 
While suggesting the emphatically empirical hypothesis that the FLN contains the ability of recursion or is identical to it, they emphasize the possibility that the FLN could in fact be just the unique, human-specific combination of the various neither human nor language specific components of FLB. In my opinion, this possibility should be taken seriously, especially since we do not know of a gene or hereditary condition that would result in an (otherwise) fully formed human linguistic capacity lacking recursion. (On Daniel Everett's view of Pirahã, see, in particular, the careful and detailed rebuttal of Nevins et al. 2009a;b.)

We know that recursion in a general sense is not a human-specific characteristic. Recursive properties of the songs of various songbirds have often been described. Berwick et al. (2012) thought that the recursion of birdsong had nothing to do with the recursion of human syntax, that it was rather analogous to aspects of human speech, that is, the sensorymotor component of human language. While this assumption is perfectly compatible with the point that recursion is not human specific, we might wonder if in addition a stronger connection might exist between recursion in FLN and the recursive property of the item-organizing systems of birdsong. Indeed, a year later (Miyagawa et al. 2013), Berwick himself considers birdsong grammar analogous to an aspect of narrow syntax, that is, of FLN.

The kind of recursion that is presumed to be characteristic of humans cannot be produced by a finite state machine, it needs at least a contextfree grammar. This type of recursion, which involves center embedding, where the output of one of two adjacent steps of recursion envelops the other, can be considered as the core or exclusive content of the FLN by Hauser et al. and Fitch et al.

Chomsky proved already in the '50s of the last century that the structure of natural language sentences cannot be generated by a finite state grammar even in terms of weak generative capacity. In other words not even the set of output strings can be generated by a finite state grammar since this set contains centrally embedded recursive structures. But even if we were to force the word-strings of natural language sentences into a finite state grammar output on some dubious grounds like the finiteness of the set of sentences that can be uttered in a lifetime, using a finite state grammar we would not be able to account for the structure of these sentences. The indisputable fact, explicitly or implicitly supported by study after study in the vast generative literature, remains that generating the structures of natural language sentences minimally requires a context-free grammar. 
Nonetheless, I would like to put forward the hypothesis that a finitestate grammar that is adequate to describe birdsongs is not only sufficient but even potentially too strong to describe the syntax of human language in the narrow sense. Of course, I do not think that a finite-state grammar is enough to describe the sentence structures of natural language, but the two concepts, 'syntax in the narrow sense' and 'sentence structures' do not necessarily coincide.

The traditional notion that form and meaning are connected through syntax is expressed also in most variants of generative grammar by a model where the syntactic structures are mapped to a sensory-motor and a conceptual-intentional interface. I assume that the structure of the sentence is a composite of several syntactic structures on the conceptualintentional interface, that syntax in the narrow sense generates only segments of the entire sentence structure. At this general level of discussion, the statement is similar to Chomsky's phase-based approach, where also proper substructures of the full sentence structure are mapped separately onto the interface. The major relevant differences are twofold. First the substructures to be generated by the respective syntaxes of the two approaches are radically different, and second, I assume that the substructures join to form a sentence structure only at the interface(s).

The essence of my proposal is that the task of the syntax in the narrow sense is to produce the paths of the sentence that lead from the initial symbol of a standard syntactic tree to each of the terminal elements. In the simple abstract syntactic tree structure (1), where $S$ is the initial symbol of the tree and the terminal elements are $a, c$ and $d$, we have the three paths listed in (2):

(1)

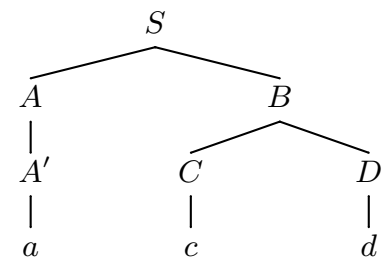

(2) $S, A, A^{\prime} a ; S, B, C, c ; S, B, D, d$

The task of the syntax is to create these paths, to create simple linear sequences. On one version of the theory the order of the elements is partly regulated by the sequence of functional categories uncovered by linguisticcartographic research (see e.g., Rizzi 1997; 2004; Cinque 1999) and partly by the simple rule that the concatenation of a legitimate path or its ter- 
minal segment to the initial segment of another legitimate path, results in a(nother) legitimate path.

For example, if $d$ in (1) is a verb, then $S, B, D, d$ can be the functional sequence of the verb. This and the fact that the sequence $S, B, D, d$ starts with the initial symbol and ends with a terminal element, the verb, makes this sequence a legitimate path. If a is a noun, the subject of the verb, then $A, A^{\prime}, a$, is the functional sequence of the noun. Concatenating this to the initial segment of the verb's path, here consisting of a single element only, $S$ (of $S, B, D, d$ ), results in another legitimate path: $S, A, A^{\prime}, a$.

My assumption is that the novelty characteristic of human language is not in the area of narrow syntax or FLN. Here, if my approach is correct, birds, with their finite state song containing complicated looping structures may even be more advanced than humans, whose finite state FLN generates only simple linear orders. The grammatical novelty lies in the ability of the human conceptual-intentional interface to simultaneously process a whole set of linear sequences with a common starting element, each created by syntax in the narrow sense. This set provides the structure of the sentence, where the interface views a category together with all the constituents that this category precedes in any one of the linear sequences a constituent. If the terminal elements are constituents, then in (1) $C$ and $c$ form a constituent, as do $D$ and $d$, and $B, C, c, D, d$ is a constituent, and so on.

This approach elegantly explains some of the central phenomena of universal grammar, which so far only had more or less stipulative accounts. It entails the property of structure dependence that follows also from the standard theory. In addition it helps to understand also the strict binary branching of the sentence structure trees, the necessity of c-command and the correlation of movement-transformations on one hand and of the dominance relations on the other with the linear order of the words, as well as aspects of the mirror principle or the lack of tree internal syntactic sideward relations (Brody 2015; 2018a;b; 2019).

It is important to ascertain also that center embedding recursion is not problematic given our restrictive view of syntax as involving only linear orders generable by a finite state system. Being able to generate infinitely large center embedding structures is of course not a novelty in the sense that these can be handled by all current context free approaches. But none of these produce FLN structures that can be generated by a finite state system. They all implicitly assume or allow the claim that an FLB that incorporates an FLN whose structures are generable by a finite state system cannot reasonably account for the (assumed infinite) center embedding property of natural language. It is I believe a step forward to be able to show that this claim can be validly rejected. 
To make sure, let us take for example, the recursive structure in (3).

(3) a. $a_{1},\left(a_{2},\left(\ldots a_{n}, b_{n}, \ldots\right) b_{2},\right) b_{1}$

b. $S$

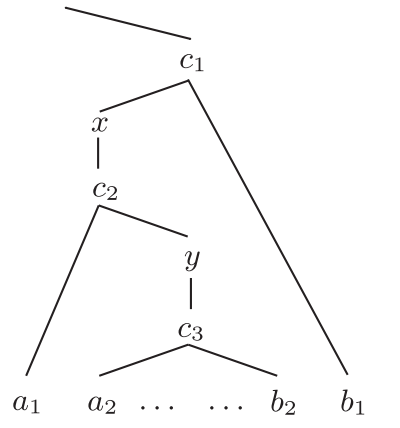

For the sake of this illustration I assume that the PF component spells out the $b$ elements to the right of their sister in the interface tree, and the a elements to the left, and also that PF cannot create crossed tree branches. The sister ordering statements are just usual cases of directionality parameter setting, and the no crossing assumption follows from the appropriate construal of the precedence relation in the linear orders, see Brody (2018a;b). (3) can then be generated as follows.

I. The category $b_{1}$ is led by some path to the starting symbol $S$, call this path $B_{1}$.

(4) $B_{1}: S, \ldots, c_{1}, \ldots b_{1}$

(5) $A_{1}: x, \ldots, c_{2}, \ldots a_{1}$

The path $A_{1}$ ending in $a_{1}$ is concatenated to the initial segment ending with $c_{1}$ of $B_{1}$. The connection point $c_{1}$ now linearly precedes $b_{1}$ (in $B_{1}$ ) and $a_{1}$ (in the newly created path), so $a_{1}, b_{1}$ will be members of the constituent $c_{1}$ at the interface.

II. Call $A_{1}{ }^{*}$ the path beginning with $S$ and ending with $a_{1}$.

(6) $A_{1}^{*}: S, \ldots, c_{1}, x, \ldots, c_{2}, \ldots, a_{1}$

To an initial segment of $A_{1}$ * that is long enough to include also an initial segment of $A_{1}$, we concatenate (at $c_{2}$ ) $B_{2}$, the path of which $b_{2}$ is the terminal element. Call $B_{2}{ }^{*}$ the path that leads from $S$ to $b_{2}$. 
(7)

$B_{2}: y, \ldots, c_{3}, \ldots, b_{2}$

(8) $B_{2} *: S, \ldots, c_{1}, x, \ldots, c_{2}, y, \ldots, c_{3}, \ldots, b_{2}$

To an initial segment of $B_{2}{ }^{*}$ that is long enough to include an initial segment of $B_{2}$ we concatenate (at $c_{3}$ ) $A_{2}$, the path ending in $a_{2}$. The connection point, $c_{3}$, precedes $a_{2}$ and $b_{2}$, which are therefore members of the constituent $c_{3}$ at the interface. Now $c_{1}$ precedes not only $a_{1}$ and $b_{1}$, but also $c_{3}$, hence $a_{1}, c_{3}$, and $b_{1}$ are members of the constituent $c_{1}$ at the interface. We have generated $\left(a_{1}\left(a_{2} b_{2}\right) b_{1}\right)$.

III. We repeat the previous step with the appropriate changes: Call $A_{2}{ }^{*}$ the path starting with $S$ and terminating with $a_{2}$. To an initial segment of $A_{2} *$ that is long enough to contain an initial segment of $A_{2}$ we concatenate the, $B_{3}$, the path whose terminal is $b_{3}$. Call $B_{3}{ }^{*}$ the path leading from $S$ to $b_{3}$. To an initial segment of $B_{3}{ }^{*}$ that is long enough to contain an initial segment of $B_{3}$ concatenate $A_{3}$, the path ending in $a_{3}$. We repeat step III mutatis mutandis, until we reach $b_{n}$ and $a_{n}$. We then have generated the set of paths, the totality of which results in the constituent structure (3) at the interface.

We have generated the structure in (3) by always concatenating the path of $a_{i}$ directly to the path of $b_{i}$ - in narrow syntax we did not need to make use of a context free grammar. No push-down (or any other type of) memory has been exploited in FLN. We obtain the center-embedding structure that only a context free grammar can produce directly, only at the interface, by using a simple and independently necessary definition of constituents.

If our approach proves to be tenable, this raises the possibility that the item-organizing specificity of human language is not due to a qualitative change and unique development in syntactic ability. It is instead a consequence of the possession of a, presumably dedicated, interpretive module which is capable of parallel processing of numerous simple one-dimensional syntactic structures. Such a component may not be more powerful or radically different from the one that interprets standard tree structures, and is needed in any case. 


\section{References}

Attwood, Tony. 2007. The complete guide to Asperger's Syndrome. Philadelphia: Jessica Kingsley.

Berwick, Robert C., Gabriel J. L. Beckers, Kazuo Okanoya and Johan J. Bolhuis. 2012. A bird's eye view of human language evolution. Frontiers in Evolutionary Neuroscience 4 (Article 5). 1-25.

Brody, Michael. 1987. On Chomsky's Knowledge of Language. Mind and Language 2. $165-177$.

Brody, Michael. 1992. Three theories of the organization of the grammar. UCL Working Papers in Linguistics 4. 1-10.

Brody, Michael. 1993. $\theta$-theory and arguments. Linguistic Inquiry 24. 1-23.

Brody, Michael. 2015. One-dimensional syntax. Manuscript. http://ling.auf.net/lingbuzz/002863.

Brody, Michael. 2018a. 'Movement', precedence, c-command. Manuscript. http://ling.auf.net/lingbuzz/004044.

Brody, Michael. 2018b. Two advantages of precedence syntax. In H. Bartos, M. den Dikken, Z. Bánréti and T. Váradi (eds.) Boundaries crossed, at the interfaces of morphosyntax, phonology, pragmatics and semantics. Chem: Springer. 319-326.

Brody, Michael. 2019. Some problems with merge. Manuscript. UCL.

Chomsky, Noam. 1995. The minimalist program. Cambridge, MA: MIT Press.

Cinque, Guglielmo. 1999. Adverbs and functional heads: A cross-linguistic perspective. Oxford: Oxford University Press.

Dékány, Éva. 2019. Foundations of generative linguistics. Acta Linguistica Hungarica 66. 309-334.

Fedor, Anna, Péter Ittzés and Eörs Szathmáry. 2009. The biological background of syntax evolution. In D. Bickerton and E. Szathmáry (eds.) Biological foundations and origin of syntax. Cambridge, MA: MIT Press. 15-40.

Fedor, Anna, Péter Ittzés and Eörs Szathmáry. 2010. A nyelv evolúciójának biológiai háttere [The biological background of language evolution]. Magyar Tudomány 171. $541-548$.

Fitch, W. Tecumseh, Marc D. Hauser and Noam Chomsky. 2005. The evolution of the language faculty: Clarifications and implications. Cognition 97. 179-210.

Hauser, Marc D., Noam Chomsky and W. Tecumseh Fitch. 2002. The faculty of language: What is it, who has it, and how did it evolve? Science 298. 1569-1579.

Karmiloff-Smith, Annette. 2006. The tortuous route from genes to behavior: A neuroconstructivist approach. Cognitive, Affective \& Behavioral Neuroscience 6. 9-17.

Kenesei, István (ed.). 2019. Nyelv, biológia, szabadság. A 90 éves Chomsky jelentősége a tudományban és azon túl [Language, biology, freedom. The importance of the 90year-old Chomsky in science and beyond]. Budapest: Gondolat.

Miyagawa, Shigeru, Robert C. Berwick and Kazuo Okanoya. 2013. The emergence of hierarchical structure in human language. Frontiers in Psychology 4. Article 71.

Nevins, Andrew, David Pesetsky and Cilene Rodrigues. 2009a. Evidence and argumentation: A reply to Everett. Language 85. 671-681. 
Nevins, Andrew, David Pesetsky and Cilene Rodrigues. 2009b. Pirahã exceptionality: A reassessment. Language 85. 355-404.

Rizzi, Luigi. 1997. The fine structure of the left periphery. In L. Haegeman (ed.) Elements of grammar. Dordrecht: Kluwer. 281-337.

Rizzi, Luigi. 2004. Locality and left periphery. In A. Belletti (ed.) Structures and beyond: The cartography of syntactic structures 3. Oxford: Oxford University Press. 223-251.

Szathmáry, Eörs. 2001. Origin of the human language faculty: The language amoeba hypothesis. In J. Trabant and S. Ward (eds.) New essays on the origin of language. Berlin \& New York: Mouton de Gruyter. 41-51.

Szathmáry, Eörs. 2002. Az emberi nyelvkészség eredete és a „nyelvi amóba” [Origin of the human language faculty: The language amoeba hypothesis]. Magyar Tudomány 108. $42-50$.

Szathmáry, Eörs. 2018. A nyelvkészség és az evolúció [The language faculty and evolution]. Paper presented at Chomsky 90: konferencia az idén 90 éves Noam Chomsky hatásáról.

Vicker, Beverly. 2009. Social communication and language characteristics associated with high functioning, verbal children and adults with autism spectrum disorder. Bloomington, IN: Indiana Resource Center for Autism. 
